\title{
Hospice Care, Disease Burden Prediction, and the "Unbefriended" Patient
}

\author{
Sayan Biswas ${ }^{1}$ \\ ${ }^{1}$ Medlounge, Kolkata, West Bengal, India
}

\begin{abstract}
Address for correspondence Sayan Biswas, BS, PGDipl CS, MedLounge, FI CC-704, Ashabari Housing Complex, Block O, BP Township, Kolkata 700094, West Bengal, India (e-mail: sayanb2011@gmail.com).
\end{abstract}

\begin{abstract}
Keywords

- unbefriended

- chronic medical condition

- comorbidity

- cognitive decline

- resource allocation

The COVID-19 pandemic has created a global mayhem. Patient care has been severely affected. Patients with chronic conditions such as dialysis-dependent end stage renal diseases are finding it challenging to seek out continuous treatment. Ground breaking work by Dr. Ravikanth Yalamuri of Methodist TexSan Hospital is paving new pathways for tackling patient volume in hospital settings, especially in hospices. In particular, Dr. Yalamuri has provided succinct insights into empathetic caring for the unbefriended patients. These elderly orphans have major issues with cognition and require high levels of social aids and support, especially when they are admitted to the hospital or when they have a terminal illness. Dr. Yalamuri has highlighted the utility of operations research in these multifaceted disease model prediction.
\end{abstract}

In the changing landscape of a highly stochastic health care environment, physicians across the globe are putting their best thoughts forward. In order to protect humanity, the current health care crisis needs fresh outlook to tackle the unprecedented levels of an extremely chaotic situation of health care delivery. Hospitals across the world have to tune up to the economy and become coherent with the complexity to operate efficiently. The aging population poses a huge burden of chronic diseases, and efficient methods of disease prevention, diagnosis, treatment planning, and proactive care are required. The modus operandi of health care systems are changing at rapid pace and current environment demands newer models of health care like telemedicine and home health care. One big challenge the current environment has posed are for patients with compromised kidney functions and requiring dialysis. The complexity has been escalated by the suppressed immune function of these patients with compromised renal functions and their propensity to codevelop the reported new infection by the SARS-Co-V-2 (COVID-19) virus. One of the pioneering work that attracted my attention is by Dr. Ravikanth Yalamuri, hospitalist of Methodist Hospital Texsan in San Antonio, Texas, United States, and Medical Director of multiple skilled nursing facilities including Buena Vida, Hunters Pond, and River City Rehabilitation Centers (-Fig. 1).

published online

September 22, 2020
DOI https://doi.org/

$10.1055 / \mathrm{s}-0040-1716803$

ISSN $2455-7420$.
Dr. Yalamuri is a MBBS from Guntur Medical College. He accomplished his residency training from the United States and the United Kingdom. Dr. Yalamuri has original scholarly work to his credit in which he examined unique methods like ureteric grafts to maintain the patency of arteriovenous fistula, the main stead for performing renal dialysis. These fistulae which are extremely important for conduction of renal dialysis, often become nonfunctional and create enormous trouble for the patients. Coupled with these are the propensities to acquire infections, including COVID-19. Recently, Dr. Yalamuri has exposited, utilizing the concepts of operations research, using principles of minimalization and optimization to enhance and functionalize renal dialysis units without interruption.

Dr. Yalamuri elaborated the scope of the problem. He mentioned, "As we know, there are impeding challenges to healthcare delivery these days. One cohort of highly vulnerable patients includes who require renal replacement therapy, otherwise known as dialysis. Many other patients require regular visits to the hospital or the ambulatory clinic for routine check-up or exacerbation of their chronic conditions. For example, a chronic heart failure patient with highly comprised ejection fraction shall require periodic evaluation of an implantable device like chronic resynchronization therapy."

(C) 2020. Medical and Surgical Update Society.

This is an open access article published by Thieme under the terms of the Creative Commons Attribution-NonDerivative-NonCommercial-License, permitting copying and reproduction so long as the original work is given appropriate credit. Contents may not be used for commercial purposes, or adapted, remixed, transformed or built upon. (https://creativecommons.org/licenses/by-nc-nd/4.0/)

Thieme Medical and Scientific Publishers Pvt. Ltd., A-12, 2nd Floor, Sector 2, Noida-201301 UP, India 


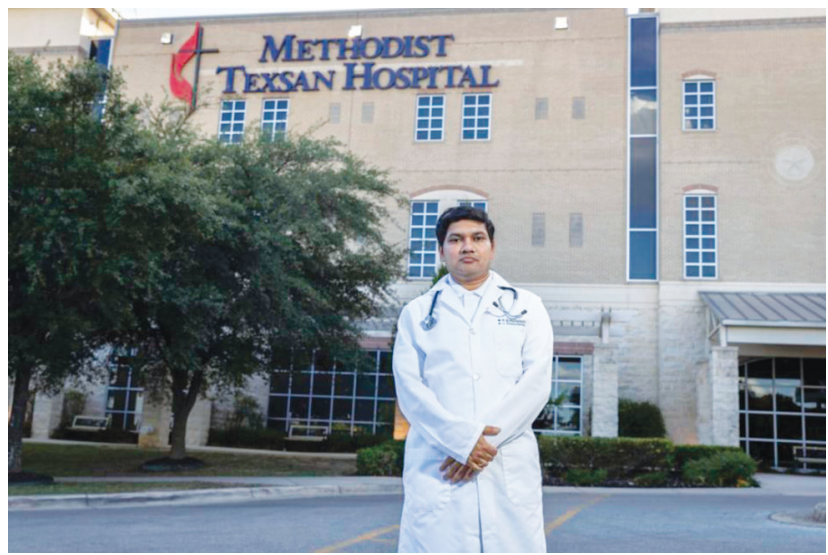

Fig. 1 Photograph of Dr. Ravikanth Yalamuri.

One of the unique ways to tackle these issues includes to estimate the burden of the health care. Dr. Yalamuri has added value to the flow by bringing in concepts of operations research to helping making an estimate of patient loads on a health care system, without allowing creeping in Berksonian bias. These prediction models were made by utilizing discrete event simulation. These methods reflect the random dynamics of a capacity constraint system. Another major factor is the ability to transport patients between their homes or hospice and an appropriate delivery system like dedicated dialysis unit. The travel times may be estimated by methods like "routino" and using Google street map. Iterated local search methods facilitate finding optimal solutions for grouping and estimating patient selection. Python and Mathlab can perform these analyses efficiently, outlined Dr. Yalamuri. These methods are generalizable to various population sets. Just like in a flu season when we estimate the disease burden, it is highly important currently to know how many prospective COVID-19 patients will turn up. This is true not only for Dr. Yalamuri's hospital but for hospitals across the globe, expressed Dr. Yalamuri. This also helps us to design informed decisions on the scale of patient discharges. Given the significant importance of social distancing, the environment in the hospice merits fine tuning on its optimization.

Patients on essential renal replacement therapy rely on essential treatment and need to travel to treatment centers. These results in considerable increase in the requirement for nonemergency ambulance transport. Dr. Yalamuri's analytical approaches have helped to assess the service capacity and create a near seamless transition between COVID-19 positive and negative patients as they travel through treatment centers. This shall also help in rapid mobilization in resources to scale up and down as the requirement fluctuates. In this fashion patients are sequestered based on their positivity and help reduce transmission of a blood-borne infection during hemodialysis. The planning for the demand is enormously helpful for operational success. Further, it is not only useful for the ambulatory clinic or dialysis center, but it is also enormously helpful for burden assessment for other practice settings like in a hospital or a hospice.
The biggest class of challenging subjects in the current scenario is the unbefriended individuals, pointed out by Dr. Yalamuri. These include the lonely elderly, homeless individuals, and the incarcerated patients. It is a massive challenge to systematically reach out to these vulnerable groups of population. It is common knowledge that they are often missed out on appropriate health care delivery options. For example, in a hospice many of these individuals do not have any visitors a few weeks or months after their initial registration. These create enormous problem in efficient communication and health care delivery. For example, consider the case of the demented patients. Many unbefriended patients have significant mental illness. Some individuals have alienated their families or may simply outlive them. There are rising numbers of unmarried or childless individuals, resulting in nearly one-fifth of seniors becoming "elder orphans." These patients often do not have a living will or advance directives, resulting in unnecessary painful treatments of which they may not be at all desirable.

Providing care to the unbefriended patients is a very difficult point. Surrogate decision makers are needed. Social workers or hospital ethics committee often play important roles for making decisions on their behalf, mentioned Dr. Yalamuri. These voiceless or vulnerable patients must raise their standards of life or to die peacefully, surrounded by people.

As a hospitalist, Dr. Yalamuri is in a unique position to help these most challenging patients. These unrepresented patients pose a predicament for hospitalists, but physicians such as Dr. Yalamuri are most uniquely poised to provide care for these patients. Often these patients have substance use disorders. As the population ages and estrangement from family members becomes the norm, numbers of this population are on the rise. An earlier study had demonstrated that nearly $5 \%$ of patients who died in the intensive care unit (ICU) did not have a surrogate decision maker. In certain states in the United States, the law allows the patients' physician to make a decision on their behalf. Usually a team effort to arise at a decision is an efficient way to provide the treatment options in the best interest of the patients. Hospitalist or physicians caring for their patients can initiate discussions and assist patients who have high risk of becoming unrepresented. This is particularly important when the subjects still have high level of cognitive functioning. Dr. Yalamuri pointed out additional avenues of their ability to interface with the hospital leadership and highlight the perspective, which is a pressing need and a national challenge.

Hospice was originally popularized by Dame Cicely Saunders, who was a social worker, nurse, and physician all in one. Hospice has now emerged as philosophy of care for individuals with varied terminal illnesses. In the current challenging health care climate, Dr. Yalamuri has been a vigorous advocate of directly discharging patients from hospital and robust support in a home health care environment. Despite the overlapping issues of common endpoint which is death, the patterns of terminal illnesses are quite different. Chronic obstructive pulmonary disease, cancer, and dementia patients 
will not die in the same fashion. Very important aspect is pain control; it is also imperative to have a transformative vision to meet the challenges of the changing times. Another aspect is that cancer patients will be immunosuppressed and are prone to infections. The spread of cancer brings in the inflection point in the life of the patient, where the illness trajectory takes a steep downward course. These critically ill patients may suddenly face death. This is a time where hospice care is merited. Sometimes if cancer treatment proves ineffective, hospice care may be needed earlier. Dr. Yalamuri have paid special attention to the management of chronic care conditions including emphasis on smoking cessation, and adherence to diabetes and blood pressure medication. He discusses goals and expectations early in the course of the disease. Having defined advance directives early in the course of illness is a plus. Individuals with lung and heart disease require more frequent hospitalization toward the end phase of their illness and may require intensive ICU intervention. Prediction modeling is very useful for ICU burden load modeling, which is so critical, given the current respiratory illness pandemic. Dr. Yalamuri's emphasis on regimented primary care is an avenue to follow for medical care of the elderly and disabled in view of the multiple challenges posed by the current health care cataclysm.

\section{Conflict of Interest}

None declared. 